\title{
Metastatic Endogenous Endophthalmitis: A Rare Presentation with Methicillin-resistant Staphylococcus aureus Prostatic Abscess
}

\author{
Mrugank M. Bhavsar, T. V. Devarajan, P. Senthur Nembi', Nagarajan Ramakrishnan², Ashwin K. Mani \\ Departments of Critical Care Medicine and ${ }^{1}$ Infectious Diseases, Apollo First Med Hospital, ${ }^{2}$ Department of Critical Care Medicine, Apollo Hospitals, \\ Chennai, Tamil Nadu, India
}

\section{Abstract}

A 62-year-old male with the previous history of uncontrolled diabetes and hypertension on irregular treatment presented with a history of fever, dysuria, and urinary retention with progressive painful loss of vision over a period of 2 days. His eye examination showed hypopyon, and he was diagnosed to have rapidly progressive endogenous endophthalmitis. He was started on vancomycin and piperacillin-tazobactam empirically. His blood and urine cultures grew methicillin-resistant Staphylococcus aureus (MRSA). Transesophageal echocardiography ruled out infective endocarditis. Intravitreal injection of vancomycin and ceftazidime was given. Vitreous culture also grew MRSA. A workup for possible source revealed multiple prostatic abscesses on the transrectal ultrasound. Antibiotic was changed to daptomycin in view of high vancomycin minimum inhibitory concentration. His vision was improved at the time of discharge.

Keywords: Endophthalmitis, methicillin-resistant Staphylococcus aureus, prostatic abscess, transrectal ultrasound, vancomycin

\section{INTRODUCTION}

Methicillin-resistant Staphylococcus aureus (MRSA) bacteremia continues to be a significant cause of morbidity and mortality in intensive care settings. The most critical determinants of survival and reduction of complications are early identification of bloodstream infection, combined with the initiation of timely and appropriate antibiotic therapy. Early recognition, prompt initiation of appropriate antibiotics, and rapid microbiological diagnosis are, therefore, key components of effective clinical management. We report a patient with a rare presentation of rapidly progressive endogenous endophthalmitis due to MRSA bacteremia secondary to prostatic abscess.

\section{Case Report}

A 62-year-old male with poorly controlled diabetes and hypertension presented with pain and decreased left eye vision for 2 days which was rapidly worsening and leading to complete loss of vision on presentation to us. Patient also had associated symptoms such as low-grade fever and dysuria for the past 1 week. On further evaluation, patient

\begin{tabular}{|l|l|}
\hline \multicolumn{2}{|c|}{ Access this article online } \\
\hline Quick Response Code: & Website: \\
\hline & www.ijccm.org \\
\hline
\end{tabular}

was noted to be febrile $\left(100.4^{\circ} \mathrm{F}\right)$, tachycardic, dehydrated, and had evidence of prostatic hypertrophy per rectal examination [Figure 2]. Local examination of the left eye revealed hypopyon with complete loss of vision (visual acuity - R/E - 6/60, L/E - only light perception). Fundoscopic examination revealed multiple vitreous exudates and findings suggestive of endogenous endophthalmitis. Preliminary investigations revealed leukocytosis (white blood cells count $14,100)$, elevated erythrocyte sedimentation rate $(48 \mathrm{~mm} / \mathrm{h})$, and poorly controlled diabetes ( $\mathrm{HbA} 1 \mathrm{C}$ of 11$)$. Urine routine showed plenty of pus cells with blood glucose level 268 . Primary diagnosis was urinary tract infection and endogenous endophthalmitis. Patient was started on piperacillin-tazobactam and vancomycin empirically with pending cultures. Urine

Address for correspondence: Dr. Ashwin K. Mani, Department of Critical Care Medicine, Apollo First Med Hospital, 154, PH Road, Chennai - 600 010, Tamil Nadu, India. E-mail: ashwin.mani@gmail.com

This is an open access article distributed under the terms of the Creative Commons Attribution-NonCommercial-ShareAlike 3.0 License, which allows others to remix, tweak, and build upon the work non-commercially, as long as the author is credited and the new creations are licensed under the identical terms.

For reprints contact: reprints@medknow.com

How to cite this article: Bhavsar MM, Devarajan TV, Nembi PS, Ramakrishnan N, Mani AK. Metastatic endogenous endophthalmitis: A rare presentation with methicillin-resistant Staphylococcus aureus prostatic abscess. Indian J Crit Care Med 2017;21:172-5. 


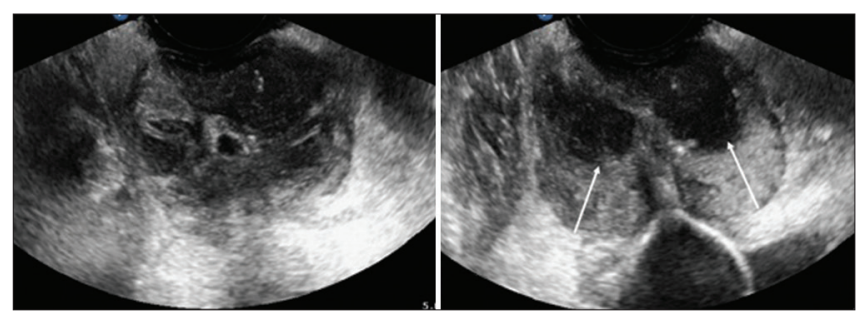

Figure 1: Prostate ultrasound showing abscess.

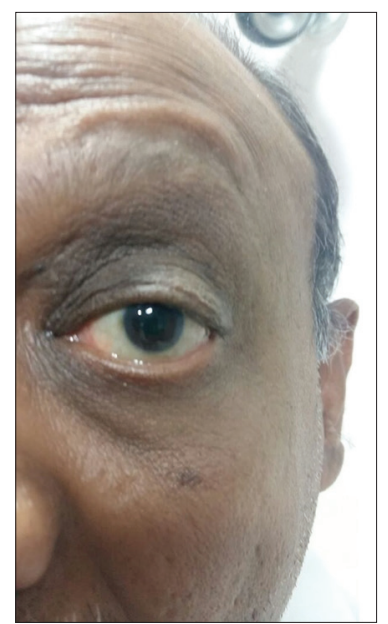

Figure 3: Left eye - after completion of treatment.

culture showed Gram-positive cocci (GPCs) and blood culture and sensitivity confirmed MRSA with vancomycin minimum inhibitory concentration (MIC) was 0.5 . Patient was continued on vancomycin. A transesophageal echocardiography revealed normal left ventricular function and no evidence of valvular disease or vegetations suggestive of endocarditis. Abdominal ultrasound showed Grade 1 prostatomegaly [Figure 1]. Transrectal ultrasound (TRUS) revealed multiple small prostatic abscesses. Urologist was consulted, and conservative management was suggested. Patient was posted for vitrectomy and intravitreal injection of antibiotics, but on the day of surgery in spite of adequate sugar control, patient's sugar level was $470 \mathrm{mg} / \mathrm{dl}$, so vitrectomy was deferred, and only intravitreal injection of vancomycin and ceftazidime was given. Vitreous fluid culture also grew MRSA. Postprocedure after 1 week, fundus examination showed subretinal abscess which was not seen in the first examination [Figure 4]. There were also features of early retinal detachment. However, the patient's vision improved to $6 / 24$ in the left eye. The bacteremia failed to clear in spite of adequate therapy with vancomycin. The repeat blood culture showed MRSA with higher MIC (vancomycin MIC >2) by E-test. Antibiotic was changed to daptomycin. After starting daptomycin, the subsequent blood cultures were negative. The patient's vision improved with antibiotics, and vitrectomy was not performed. He was discharged with daptomycin to continue for 4 weeks. At the time of discharge, patient vision was 6/60. Patient was on regular follow-up with infectious disease specialist and ophthalmologist as an outpatient, and antibiotic therapy was

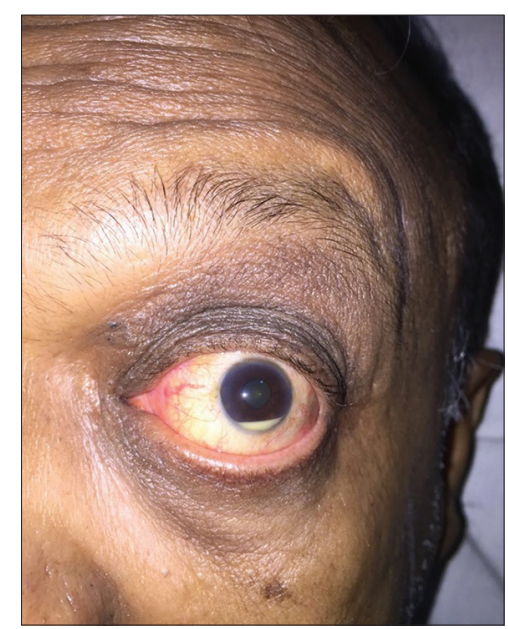

Figure 2: Left eye hypopyon.

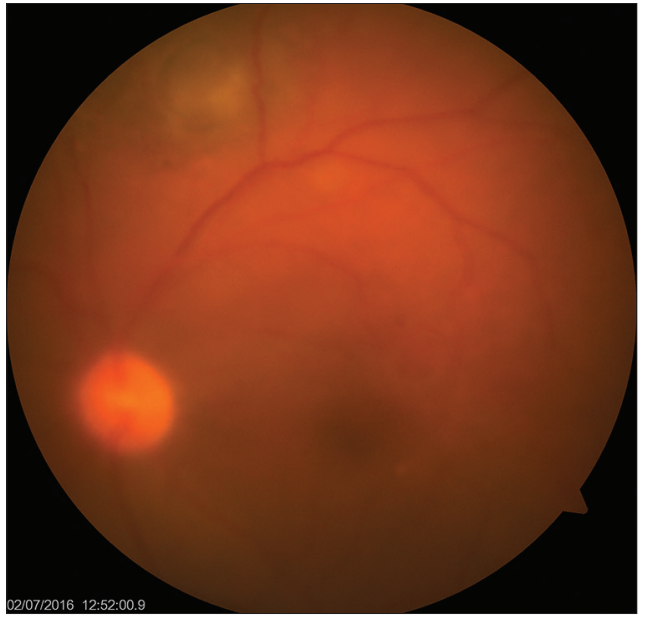

Figure 4: Fundus examination - subretinal abscess.

continued. After completion of 4-week antibiotics, ophthalmic examination showed no hypopyon [Figure 3], and there was also significant vision improvement from $6 / 60$ to $6 / 18$. Follow-up of TRUS showed resolved prostatic abscesses. Patient's urinary symptoms had completely resolved too. Urine analysis was normal, and urine cultures did not show significant growth. Once daptomycin therapy was completed, the patient was initiated on oral cotrimoxazole for which MRSA strain was sensitive and was advised to complete the course of treatment for prostatic abscess. After 2 months from discharge, patient underwent retinal reattachment surgery as a sequel of subretinal abscess with endogenous endophthalmitis [Figure 5]. Patient's current vision is 6/60 in the left eye.

\section{Discussion}

Intraocular colonization by bacteria can occur after trauma (exogenous endophthalmitis) or systemic dissemination (endogenous or metastatic endophthalmitis). Endogenous bacterial endophthalmitis is perhaps the rarest form and may be caused by organisms such as Escherichia coli, Staphylococcus aureus, and Klebsiella ${ }^{[1]}$ In 


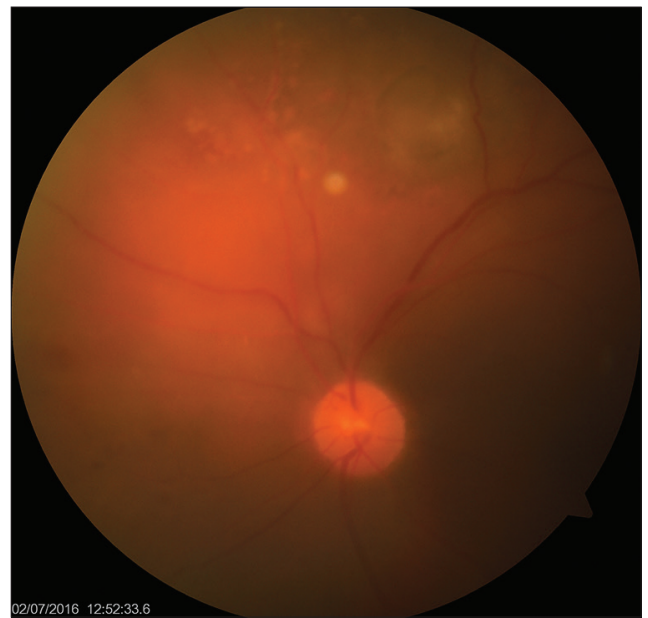

Figure 5: Resolving abscess.

an immunocompromised patient, the sources are the gastrointestinal tract (hepatic abscess and appendicitis), genitourinary tract (renal abscess and infected stones), and the heart, ear, etc. Metastatic bacterial endophthalmitis from a genitourinary source is rare. ${ }^{[2]}$

$S$. aureus bacteremia is common and increasing worldwide and is associated with substantial morbidity and mortality. It is often associated with a local focus of infection that has gained access to the bloodstream. MRSA is a significant cause of both healthcare-associated and community-acquired infections. ${ }^{[3,4]}$

The inability to rapidly identify and characterize infecting organisms means that initial antibiotic therapy is often empirical. This may result in inappropriate treatment, which is associated with extended overall duration of hospitalization, increased risk of patient mortality, and increased overall cost of treatment, particularly for patients infected with MRSA. However, with the emergence and increasing prevalence of community-associated methicillin-resistant strains, the choices of standard empirical antibiotic therapy are often reconsidered to cover resistant organisms. From a clinical standpoint, given the morbidity and mortality associated with delayed treatment of MRSA infection, it would be necessary to include MRSA coverage in empirical antibiotic regimens in settings where a significant proportion of patients hospitalized for community-acquired S. aureus infection have methicillin-resistant strains. This may of course be deescalated if culture is returned as methicillin sensitive strains (MSSA).

A recent review study demonstrated key clinical principles in the management of $S$. aureus bacteremia; all infective foci must be identified and removed as soon as possible, and long-term antimicrobial therapy is required for those with persistent bacteremia or a deep, irremovable focus. The best drugs, dose, mode of delivery, and duration of therapy remained uncertain, a situation compounded by emerging $S$. aureus strains that are resistant to old and new antibiotics. ${ }^{[5]}$

Clinical outcomes could be inferior in patients with MRSA having vancomycin MIC $>1$. Teicoplanin would not be effective in patient with higher MIC for vancomycin. Linezolid is an option, but it is not approved therapy for MRSA bacteremia and prolonged duration of therapy associated with toxicities such as cytopenia and neuropathy. Hence, daptomycin was chosen.

In our case report, our primary focus is that an unusual presentation of MRSA prostatic abscesses associated with rapidly progressing endogenous endophthalmitis should not be missed and aggressive intervention must be needed to prevent permanent loss of vision. In previous case reports, vitrectomy was done, few patients developed retinal detachment, and few patients lost vision and required enucleation. ${ }^{[6-9]}$ In our case report, patient with endogenous endophthalmitis from MRSA bacteremia was managed without vitrectomy.

New management strategies are required including the use of techniques that allow the rapid identification of $S$. aureus bacteremia which enables the administration of appropriate antibiotic therapy and the identification of new drugs that enables effective empirical treatment against both susceptible and resistant $S$. aureus.

\section{Conclusion}

Rapidly progressive endogenous endophthalmitis should lead to suspicion for bacteremia and a diligent workup for a possible source. The management of these patients can be complex and requires a multidisciplinary effort. Early diagnosis of primary foci along with metastatic spread needed to manage the patient and prevent loss of vision.

\section{Declaration of patient consent}

The authors certify that they have obtained all appropriate patient consent forms. In the form the patient(s) has/have given his/her/their consent for his/her/their images and other clinical information to be reported in the journal. The patients understand that their names and initials will not be published and due efforts will be made to conceal their identity, but anonymity cannot be guaranteed.

\section{Financial support and sponsorship}

Nil.

\section{Conflicts of interest}

There are no conflicts of interest.

\section{References}

1. Bhat S, Fassaludeen M, Thomas A, Cherian J. Metastatic bacterial endophthalmitis from a prostatic abscess. BJU Int 2003;92 Suppl 3:e21.

2. Sadiq MA, Hassan M, Agarwal A, Sarwar S, Toufeeq S, Soliman MK, et al. Endogenous endophthalmitis: Diagnosis, management, and prognosis. J Ophthalmic Inflamm Infect 2015;5:32.

3. Bader M, Hinthorn D, Bajracharya H, Desai S, Kumar V. MRSA bacteremia with prostate abscess and endophthalmitis. Infect Med 2005;22:379-81.

4. David MZ, Daum RS. Community-associated methicillin-resistant Staphylococcus aureus: Epidemiology and clinical consequences of an emerging epidemic. Clin Microbiol Rev 2010;23:616-87. 
5. Thwaites GE, Edgeworth JD, Gkrania-Klotsas E, Kirby A, Tilley R, Török ME, et al. Clinical management of Staphylococcus aureus bacteraemia. Lancet Infect Dis 2011;11:208-22.

6. Ness T, Schneider C. Endogenous endophthalmitis caused by methicillin-resistant Staphylococcus aureus (MRSA). Retina 2009;29:831-4.

7. Newman DO, Lisa A, Waller S, Fernando M, Jessica R. Endogenous invasive community acquired methicillin-resistant
Staphylococcus aureus endophthalmitis: Observations in two cases. Kans J Med 2011;7:106-10.

8. Nishida T, Ishida K, Niwa Y, Kawakami H, Mochizuki K, Ohkusu K. An eleven-year retrospective study of endogenous bacterial endophthalmitis. J Ophthalmol 2015;2015:261310.

9. Ho V, Ho LY, Ranchod TM, Drenser KA, Williams GA, Garretson BR. Endogenous methicillin-resistant Staphylococcus aureus endophthalmitis. Retina 2011;31:596-601. 